\title{
1 High-resolution cryo-EM structure of photosystem II: Effects of electron beam
}

\section{2 damage}

3 Koji Kato $^{1,4}$, Naoyuki Miyazaki ${ }^{2,4}$, Tasuku Hamaguchi ${ }^{3,4}$, Yoshiki Nakajima ${ }^{1}$, Fusamichi

4 Akita $^{1, *}$, Koji Yonekura ${ }^{3, *}$ and Jian-Ren Shen ${ }^{1, *}$

5

$6{ }^{1}$ Research Institute for Interdisciplinary Science and Graduate School of Natural

7 Science and Technology, Okayama University, 3-1-1 Tsushima Naka, Okayama

8 700-8530, Japan

$9{ }^{2}$ Life Science Center for Survival Dynamics, Tsukuba Advanced Research Alliance

10 (TARA), University of Tsukuba, Ibaraki 305-8577, Japan

$11{ }^{3}$ Biostructural Mechanism Laboratory, RIKEN Spring-8 Center, 1-1-1 Kouto, Sayo-cho,

12 Sayo-gun, Hyogo 679-5148, Japan

13

$14{ }^{4}$ These authors contributed equally to this work.

$16 *$ Corresponding Authors:

17 Fusamichi Akita, E-mail: fusamichi_a@okayama-u.ac.jp

18 Koji Yonekura, E-mail: yone @ spring8.or.jp

19 Jian-Ren Shen, E-mail: shen@cc.okayama-u.ac.jp 
24 Abstract

25 Photosystem II (PSII) plays a key role in water-splitting and oxygen evolution. X-ray

26 crystallography has revealed its atomic structure and some intermediate structures.

27 However, these structures are in the crystalline state, and its final state structure has not

28 been solved because of the low efficiencies of the S-state transitions in the crystals. Here

29 we analyzed the structure of PSII in solution at $1.95 \AA$ resolution by single-particle

30 cryo-electron microscopy (cryo-EM). The structure obtained is similar to the crystal

31 structure, but a PsbY subunit was visible in the cryo-EM structure, indicating that it

32 represents its physiological state more closely. Electron beam damage was observed at a

33 high-dose in the regions that were easily affected by redox states, which was reduced by

34 reducing the electron dose. This study will serve as a good indicator for determining

35 damage-free cryo-EM structures of not only PSII but also all biological samples,

36 especially redox-active metalloproteins. 


\section{Introduction}

40 PSII is a multi-subunit pigment-protein complex embedded in the thylakoid

41 membranes of higher plants, green algae and cyanobacteria, and is the only molecular

42 machine capable of oxidizing water by use of visible light in the nature. Water molecules

43 are split into electrons, hydrogen atoms and oxygen molecules at the catalytic center of

44 PSII, namely, the oxygen-evolving complex (OEC), through four electron and/or proton

45 removing steps as described in the Si-state cycle (with $\mathrm{i}=0-4$, where $\mathrm{i}$ indicates the

46 number of oxidative equivalents accumulated $)^{1}$.

47 In order to elucidate the mechanism of the water-splitting reaction, the structure of

48 PSII has been studied extensively by X-ray diffraction (XRD) with synchrotron radiation

$49(\mathrm{SR})^{2-6}$. The SR structure of PSII at an atomic resolution revealed that $\mathrm{OEC}$ is a $\mathrm{Mn}_{4} \mathrm{CaO}_{5}$

50 cluster organized into a distorted-chair form, in which the cuboidal part is composed of

$51 \mathrm{Mn}_{3} \mathrm{CaO}_{4}$ and the outer manganese is attached to the cuboid via two $\mu$-oxo-bridges ${ }^{6}$.

52 However, based on the extended X-ray absorption fluorescence spectra (EXAFS)

53 analysis, the dose used for collecting the SR structure at 1.9 A resolution may cause $25 \%$

54 of the $\mathrm{Mn}$ ions in OEC to be reduced to $2^{+}$ions, causing some elongations in the Mn-Mn

55 distances in the structure ${ }^{7}$. This issue is overcome by the use of X-ray free electron lasers

56 (XFEL), which provide X-ray pulses with ultra-short durations that enable collection of

57 the diffraction data before onset of the radiation damage (diffraction before destruction) ${ }^{8}$.

58 Using XFELs, radiation damage free structure of PSII was solved at a high resolution by

59 an approach called fixed-target serial rotational crystallography, which uses multiple

60 large PSII crystals by a shot-and-move/rotation method ${ }^{9,10}$. The result showed a 
61 shortening of $0.1-0.2 \AA$ in some of the Mn-Mn distances, indicating that the structure

62 represents a damage free one ${ }^{10}$. By a combination of serial femtosecond X-ray

63 crystallography (SFX) with XFELs and small crystals, structures of S-state intermediates

64 up to $S_{3}$-state were analyzed by pump-probe experiments where snapshot diffraction

65 images were collected from flash-illuminated PSII crystals ${ }^{11-14}$. These results

66 demonstrated the appearance of a new oxygen atom O6 (Ox) close to O5 between Mn1

67 and Mn4 upon two flashes, suggesting insertion of a water molecule in the $S_{2} \rightarrow S_{3}$

68 transition for $\mathrm{O}=\mathrm{O}$ bond formation. However, all these studies were conducted with PSII

69 crystals, and the efficiencies of the S-state transitions in the microcrystals were reported

70 to be slightly lower compared with those in solution using light-induced Fourier

71 transform infrared difference spectroscopy ${ }^{15}$. Moreover, it is unknown if the structure of

72 PSII in the crystalline state is the same as those in the solution.

73 Cryo-electron microscopy (cryo-EM) can solve the structures of proteins in solution

74 without crystallization, which may represent the physiological states of proteins more

75 closely. It can also analyze the dynamic changes of proteins in solutions in the time range

76 of ms, provided that cooling of the samples is rapid enough. In recent years, the technique

77 of cryo-EM has been developed rapidly, and the resolutions of structures that can be

78 solved by cryo-EM are increased dramatically ${ }^{16-18}$. However, there is also the issue of

79 damage caused by the electron beam during cryo-EM data collection, even though the

80 cryo-EM is usually conducted at a low temperature. Radiation damage has been

81 extensively studied with X-rays, and it has been shown that the damage mainly manifests

82 as breakage of disulfide bonds, decarboxylation of acidic amino and photoreduction of 
83 metal centers ${ }^{7,19-21}$. The damage caused by electron beams have also been shown in

84 cryo-EM analysis ${ }^{17}$. In order to obtain a high resolution, however, cryo-EM studies are

85 usually conducted at a high-dose of electron beams without paying much attention to the

86 electron beam damage. In this paper, we analyzed the structure of PSII in ice by cryo-EM

87 at a resolution of $1.95 \AA$, and investigated the electron beam damage to PSII, especially

88 its OEC, upon dose accumulation. We show that the structure of PSII analyzed by cro-EM

89 may represent the physiological state more closely, as it retains the PsbY subunit.

90 However, it suffers from a severe electron beam damage at a high-dose, and this damage

91 was reduced at a much decreased dose without a significant loss of resolution. These

92 results are not only important for the analysis of the PSII structure in solution, but also

93 provide important implications for all cryo-EM studies that use considerably high-doses

94 for imaging.

96 Result

97 High resolution single particle analysis of the PSII

98 To obtain the high resolution structure of PSII, three data sets of single-particle

99 images of the PSII dimer from T. vulcanus were collected using Thermo Fisher Scientific

100 Titan Krios and JEOL CRYO ARM 300 at different conditions as summarized in Table 1.

101 Because the sample for the $75 \mathrm{x} \mathrm{k}$ magnification using Titan contained $5 \%$ glycerol in the

102 buffer, the sample was diluted ten times with the buffer without glycerol. The other

103 samples did not contain the glycerol and were concentrated by PEG 1450 precipitation in

104 the final step. Image processing yielded final resolutions of $2.22 \AA$ for the data set 
105 collected at $75 \mathrm{x}$ k magnification using Titan Krios (Titan-75k), $2.20 \AA$ for the data set

106 collected at $96 \times$ k magnification using Titan Krios (Titan-96k), and $1.95 \AA$ for the data

107 set collected at $60 \mathrm{x}$ k magnification using CRYO ARM 300 (ARM-60k) (Table 1, Table

1082 and Supplementary Fig. 1-4). These results indicate that the quality of the cryo-EM

109 density maps achieved were at the level comparable to those obtained with SR and XFEL

110 previously $^{6,10}$. The resolutions of the Titan-75k and Titan-96k data were almost the same,

111 in spite of the different magnifications and buffers used. The resolution of the ARM-60k

112 data was significantly better than that of the Titan-96k data, despite that the same buffer

113 condition was used for the two data sets. The resolution achieved by cryo-EM depends on

114 a number of factors, including sample quality, the type and preparations of cryo-grids

115 used, the thickness of ice in the samples, microscope alignment and imaging conditions,

116 etc. However, the major one could be the electron beam source. The CRYO ARM 300

117 microscope has a cold field emission gun (CFEG) that produces an electron beam with a

118 high temporal-coherence and superior high-resolution signals ${ }^{22}$ over that from the

119 Schottky emission gun equipped in the Krios microscope.

120 In Fig. 1, the squared inverse resolution of reconstructions achieved from random

121 subsets of particles is plotted against the subset size on a logarithmic scale. This is known

122 as Rosenthal-Henderson plot $^{23}$. These plots indicated that the resolution is proportional to

123 the $\log$ of particle size. The B-factors estimated from these plots are 60.8 for the

124 Titan-75k data set, 74.9 for the Titan-96k data set and $43.3 \AA^{2}$ for the ARM- $60 \mathrm{k}$ data set.

125 The ARM-60k data set again shows the lowest value, in agreement with its highest 126 resolution. 


\section{Overall structure of PSII}

129 The overall atomic model of PSII was built based on the highest $1.95-\AA$ resolution

130 density map reconstructed from the ARM-60k data set. At this resolution, the features of

131 cofactors and water molecules can be easily identified in the map (Fig. 2). The overall

132 architecture of the PSII dimer from T. vulcanus is very similar to that of SR (PDB:

$1333 \mathrm{WU} 2)^{6}$ and XFEL structures (PDB: 4UB6 and 4UB8) ${ }^{10}$, except for PsbY. The density of

134 PsbY is present in one of the two monomers in the native (PDB: 4UB6) ${ }^{10}$ and the

$135 \mathrm{Sr}^{2+}$-substituted PSII dimer structures (PDB: 4IL6) ${ }^{24}$ but absent in the SR structure (PDB:

1363 WU2) ${ }^{6}$. However, this density was seen in both sides of the PSII dimer in the cryo-EM

137 structure, although the density is somewhat poorer compared with that of the other

138 assigned subunits (Fig. 2a, f). This suggests that the cryo-EM structure more closely

139 represents the native state of PSII.

140 The root mean square deviation (RMSD) is $0.40 \AA$ for $5227 \mathrm{C}_{\alpha}$ atoms between the

141 structures of cryo-EM and SR, and $0.46 \AA$ for $5267 \mathrm{C}_{\alpha}$ atoms between the structures of

142 cryo-EM and XFEL. Because the RMSD was $0.32 \AA$ for $5241 \mathrm{C}_{\alpha}$ atoms between the SR

143 and XFEL structures of PSII dimers, the cryo-EM structure is almost identical to the SR

144 and XFEL structures at the backbone level. In the cryo-EM density map, we assigned

1452432 water molecules at a contour level of $5 \sigma$, which are slightly less than the number of

146 waters assigned in the SR and XFEL structures ${ }^{6,10}$. The atomic displacement parameter

147 (ADP) of the cryo-EM structure refined with Refmac5 in reciprocal space correlated well

148 with that of the SR structure (Supplementary Fig. 5), although it may be somewhat 
149 overestimated in the cryo-EM structure. Since the cryo-EM map was subjected to

150 B-factor sharpening with Postprocessing, it is not suitable to compare ADP values

151 directly between cryo-EM and X-ray structures. Nevertheless, the relative ADP of the

152 atoms in the molecule appears to reflect the characteristics of the map. The average ADP

153 for the OEC atoms $\left(13.8 \AA^{2}\right)$ were found to be lower than that observed in the overall

154 protein atoms of the cryo-EM structure $\left(20.6 \AA^{2}\right)$, suggesting that the structure of OEC

155 was determined more reliably than that of the overall structure. This may be due to the

156 presence of metal ions in the $\mathrm{Mn}_{4} \mathrm{CaO}_{5}$ cluster, which gives rise to higher cryo-EM

157 density than that of lighter protein atoms.

159 Electron beam damage to the PSII structure

160 Several regions of PSII were found to have different structures between cryo-EM

161 and XFEL, which are considered to arise from electron beam induced damage. In the

162 PsbO subunit, a disulfide bond between Cys19 and Csy41 was observed in the XFEL

163 structure $^{10}$, but it was completely cleaved in the cryo-EM structure (Fig. 3a). In the C

164 terminus of D1 subunit, a part of Ala344, the C-terminal residue that coordinated to the

$165 \mathrm{Mn}_{4} \mathrm{CaO}_{5}$ cluster, flipped out from the OEC and adopted an alternative conformation in

166 the cryo-EM structure (Fig. 3b). These are the typical sign of damage caused by the

167 electron beam irradiation during the acquirement of cryo-EM images.

168 In the OEC, the positions of heavy metals were confirmed clearly and were assigned

169 based on their highest peaks in the cryo-EM map achieved at the high-dose (Fig. 4a). In

170 addition, five oxo-oxygen atoms and four water molecules ligated to the OEC were 
171 assigned in the difference map, which were obtained by subtracting the metal densities in

172 a diameter of $1.5 \AA$ of that metal from the whole cryo-EM map. The overall architecture

173 of the OEC in the cryo-EM structure is very similar to that of the SR and XFEL structures,

174 however, distinct differences were observed in Mn-Mn and Mn-O distances (Table 3).

175 The Mn-Mn distances calculated from the initially assigned positions based on the

176 cryo-EM density were $2.8 \AA$ for Mn1-Mn2, $3.5 \AA$ for Mn1-Mn3, $5.0 \AA$ for Mn1-Mn4,

177 3.1 $\mathrm{A}$ for Mn2-Mn3, $5.4 \AA$ for Mn2-Mn4, and 2.7 ̊ for Mn3-Mn4 (Table 3). Except the

$178 \mathrm{Mn} 1-\mathrm{Mn} 4$ and Mn3-Mn4 distances, all of the Mn-Mn distances are 0.1-0.2 $\AA$ and 0.1-0.4

$179 \AA$ A longer than those of the SR and XFEL structures, respectively ${ }^{6,10}$. Most of the Mn-O

180 distances in the cryo-EM structure were also $0.1-0.5 \AA$ and $0.1-0.7 \AA$ longer than those in

181 the SR and XFEL structures, respectively (Table 3) ${ }^{6,10}$. These differences may be caused

182 by two factors. One is the electron beam damage, and the other one is the experimental

183 errors in determining the positions of the individual atoms based on the cryo-EM map

184 only. Especially, the position of oxygen atoms may not be determined precisely because

185 the map of the oxygen atoms cannot be separated from the map of metal atoms. Thus, the

186 OEC structure was refined with the restraints for bond distances $(\mathrm{Mn}-\mathrm{O}$ and $\mathrm{Ca}-\mathrm{O})$ that

187 were taken from the initial positions. The Mn-Mn distances refined with restraints were

$1882.8 \AA$ for Mn1-Mn2, $3.4 \AA$ for Mn1-Mn3, $5.0 \AA$ for Mn1-Mn4, $3.1 \AA$ for Mn2-Mn3, 5.6

$189 \AA$ for Mn2-Mn4, and $3.0 \AA$ for Mn3-Mn4 (Fig. 4b and Table 3). Except the Mn1-Mn4

190 distance, all of the Mn-Mn distances are still 0.1-0.2 $\AA$ and 0.1-0.4 $\AA$ longer than those of

191 the SR and XFEL structures, respectively ${ }^{6,10}$. Most of the Mn-O distances in the cryo-EM

192 structure refined with the restraints were also 0.1-0.4 $\AA$ longer than those in the SR and 
193 XFEL structures (Table 3). In addition, the occupancy of the OEC atoms refined with

194 Refmac5 were found to be lower than $1.0(0.87)$. These results indicate that the OEC is

195 reduced by electron dose exposed, leading to the elongation of the Mn-Mn and Mn-O

196 distances and some disorder or displacement of the metal centers during the cryo-EM

197 data acquisition. This reduced occupancy is in agreement with the previous theoretical

198 calculation of the cryo-EM structure of higher plant PSII-LHCII supercomplex ${ }^{25}$, which

199 may be the reason why a part of Ala344 flipped out and does not ligate to the OEC. The

200 reduction of metal ions with electron doses was already observed in electron

201 crystallography previously ${ }^{26}$.

202 Further structural changes in the redox-active sites, including reaction center

203 chlorophylls, electron transfer chain, proton channels and water channels, were not found

204 except the water molecule near D2-Tyr160 ( $\left.Y_{D}\right)$ (Supplementary Fig. 6c). This water

205 molecule was disordered at two positions with one being able to hydrogen bond to $Y_{D}$ and

206 the other one being able to hydrogen bond to D2-Arg180 in the SR structure and XFEL

207 structures ${ }^{6,10}$. In the cryo-EM structure, this water molecule was ordered and connected to

208 D2-Arg180. This may reflect the electron beam induced damage, which causes reduction

209 of $\mathrm{Y}_{\mathrm{D}}$ and broken the hydrogen-bond to $\mathrm{Y}_{\mathrm{D}}^{+}$.

211 Reducing electron beam dosage in determining the PSII structure

212 In order to reduce the electron beam damage, the final cryo-EM maps were

213 calculated from only initial several frames of each movie stack. In Supplementary Fig. 7,

214 the inverse resolutions of reconstructions achieved from decreased electron doses for the 
215 ARM-60k data set, associated with the frame numbers, are plotted against the dose values

216 on a logarithmic scale. Surprisingly, the electron doses from $83 \mathrm{e}^{-} \AA^{-2}$ down to $10 \mathrm{e}^{-} \AA^{-2}$

217 gave rise to almost the same resolution, indicating that increase in the electron beam

218 dosage during this range does not contribute to increase in the resolution significantly.

219 Near atomic resolution is retained at the total dose of $3.3 \mathrm{e}^{-} \AA^{-2}$ for the ARM-60k data set

220 (2.08 $\mathrm{A})$, which was achieved by using the initial 2 frames of each micrograph.

221 An overall atomic model of the low-dose PSII was built based on the highest $2.08 \AA$

222 resolution density reconstructed from the ARM-60k data set at the dose of $3.3 \mathrm{e}^{-} \AA^{-2}$

223 (Table 2). The overall architecture of the low-dose PSII is very similar to that of the

224 high-dose PSII, with a RMSD of $0.21 \AA$ for $5310 \mathrm{C}_{\alpha}$ atoms between the structures of

225 high-dose and low-dose. However, in the regions where structural changes were observed

226 due to electron beam damage, the disulfide bond between Cys19 and Csy41 of the PsbO

227 was restored at a dose of $5 \mathrm{e}^{-} \AA^{-2}$, and Ala344 of the D1 subunit was returned to the single

228 conformation to ligate to the OEC similar to those seen in the crystal structures (Fig. 3, 4

229 and 5, Supplementary Fig. 6 and 8). The ADP for the OEC atoms $\left(12.8 \AA^{2}\right)$ were lower

230 than that observed in the overall protein atoms of the cryo-EM structure $\left(22.4 \AA^{2}\right)$, and the

231 occupancy value of the OEC atoms refined with Refmac5 was returned to 1.0. These

232 results indicate the reduction of the electron beam damage in the structure. However,

233 similar to the high-dose structure, the water molecule near $Y_{D}$ are connected to

234 D2-Arg180 in an ordered manner and not hydrogen-bonded to $Y_{D}$ (Supplementary Fig. 6),

235 indicating that some electron beam damage remained.

236 The Mn-Mn distances calculated from the initially assigned positions based on the 
cryo-EM density were $3.0 \AA$ for Mn1-Mn2, 3.4 ̊̊ for Mn1-Mn3, 5.3 $\AA$ for Mn1-Mn4,

$2382.8 \AA$ for Mn2-Mn3, 5.4 ̊ for Mn2-Mn4, and 3.1 ̊̊ for Mn3-Mn4 (Table 3). All of these

239 Mn-Mn distances are $0.1-0.3 \AA$ longer than those of the SR and XFEL structures ${ }^{6,10}$.

240 Most of the Mn-O distances in the cryo-EM structure were also 0.1-0.6 $\mathrm{A}$ and $0.1-0.8 \AA$

241 longer than those in the SR and XFEL structures, respectively (Table 3). As is done with

242 the high-dose structure, we refined the OEC structure with the restraints for bond

243 distances of $\mathrm{Mn}-\mathrm{O}$ and $\mathrm{Ca}-\mathrm{O}$ that were taken from the initial positions. The $\mathrm{Mn}-\mathrm{Mn}$

244 distances in the OEC refined with the restraints were $2.9 \AA$ for Mn1-Mn2, $3.2 \AA$ for

$245 \mathrm{Mn} 1-\mathrm{Mn} 3,5.1 \AA$ for Mn1-Mn4, $2.9 \AA$ for Mn2-Mn3, $5.4 \AA$ for Mn2-Mn4, and $3.0 \AA$ for

246 Mn3-Mn4 (Fig. 4f, Table 3). Most of these distances are shorter than those of the

247 high-dose structure and close to those of the XFEL structure, although most of them are

248 still longer than the SR and XFEL structures by $0.1 \AA$ and $0.1-0.2 \AA$, respectively (Table

249 3). The Mn-O distances after refinement with the restraints also became close to the

250 XFEL structure, indicating the necessity of refinement with restraints. However, some of

251 the Mn-O distances were still longer or deviated from those found in the XFEL structure,

252 which may be caused by the electron beam damage remained and/or coordinate errors in

253 the cryo-EM analysis at the current resolution.

255 Discussion

256 In recent years, the resolution of single-particle cryo-EM has improved to atomic

257 resolutions without crystallization, and it has been reported that biological samples are 258 damaged by electron beams. In this study we elucidated the structure of the PSII at $1.95 \AA$ 
259 resolution in solution by cryo-EM, which is similar to the SR or XFEL structure in the

260 crystalline state except the PsbY subunit, which was visible in the cryo-EM structure but

261 absent or partially visible in the SR and XFEL structures ${ }^{6,10}$. This indicates that the

262 structure solved by cryo-EM more closely represents the physiological state.

263 Despite the total electron dose of $83 \mathrm{e}^{-} \AA^{-2}$ which is commonly used in the acquisition

264 of cryo-EM images, radiation damages are found in regions susceptible to redox changes,

265 i.e. the disulfide bond and the redox-active metals, whereas the overall structure is very

266 similar to those of the SR and XFEL structures ${ }^{6,10}$. The exposure of the sample to a flux of

267 electrons is conveniently expressed in terms of electrons per $\AA^{2}$ of specimen surface area

$268\left(\mathrm{e}^{-} \AA^{-2}\right)$, which is converted to the SI unit for the absorbed ionizing radiation, the Gray (Gy,

269 with $1 \mathrm{~Gy}=1 \mathrm{~J} \mathrm{~kg}^{-1}$ ), by a factor of $\square 3.7^{27}$. Thus, the total electron dose of $83 \mathrm{e}^{-} \AA^{-2}$ is

270 equal to the absorption of 307 MGy, which greatly exceeds the Henderson limit (20

271 MGy) that is the X-ray dose that a cryo-cooled crystal can absorb before the diffraction

272 pattern decays to half of its original intensity ${ }^{28}$. Nevertheless, our cryo-EM structure is

273 almost the same to the SR and XFEL structures in the redox-active sites, including

274 reaction center chlorophylls, electron transfer chain, proton channels and water channels,

275 indicating that the radiation damage does not affect the structure significantly. This is

276 considered to be the result of successful dose-weighted correction in the Bayesian

277 polishing step. However, in the PsbO subunit, disulfide bond between Cys 19 and Csy41

278 were completely breaking (Fig. 3) in the cryo-EM structure. In the OEC structure, the

279 Mn-Mn were 0.1-0.4 $\AA$ longer than those in the XFEL structure, and most of the Mn-O

280 distances were also significantly longer. In addition, the occupancy of the OEC atoms 
281 were lower than $1.0(0.87)$, resulting in a multiple conformation of the C-terminal residue

282 of D1, where a part of Ala344 flipped to a direction that does not ligate to the OEC.

283 We examined whether radiation damage could be reduced by reducing the total

284 number of stacked movie frames used in the structural analysis. In the electron doses

285 analyzed, the reconstructed map from summing the initial two frames of each micrograph

$286\left(3 \mathrm{e}^{-} \AA^{-2}, 11.1 \mathrm{MGy}\right)$ gave rise to almost similar resolutions to that of high-dose data set

287 (83 $\mathrm{e}^{-} \AA^{-2}, 307$ MGy) (Supplementry Fig.7). The reconstructed map from the ARM-60k

288 data set at the dose of $3.3 \mathrm{e}^{-} \AA^{-2}$ (11.1 MGy) gave a resolution of $2.08 \AA$. This structure

289 was compared with that obtained with the high-dose, and it was found that the disulfide

290 bond in the PsbO was recovered, and Ala344 of the D1 subunit was returned to the single

291 conformation similar to the SR and XFEL structures (Fig. 3 and 5). This indicates a

292 significant reduction of the electron beam damage to the structure. In the structure of

293 OEC, most of the Mn-Mn and Mn-O distances became shorter than those observed in the

294 high-dose structure before refinement. However, some of them are still significantly

295 deviated from those of the XFEL structure (Table 3), which become closer after the

296 refinement with restraints starting from the initial structure. Thus, it is advisable to refine

297 the cryo-EM structure with restraints imposed for compounds such as the $\mathrm{Mn}_{4} \mathrm{CaO}_{5}$

298 cluster.

299 After refinement, most of the Mn-Mn and Mn-O distances of the low-dose structure

300 are similar to those observed in the XFEL structure. However, some of the distances are

301 still longer than or deviated from those found in the damage free XFEL structure ${ }^{10}$. This

302 may be caused by two reasons; one is some electron beam damage remained, and the 
303 other one is coordinate errors existed in the cryo-EM structure. It has been reported that

304 about $80 \%$ of Mn of OEC in solution is reduced to divalent cations by an X-ray dose of 5

$305 \mathrm{MGy}^{7}$. Even though it is estimated that about $90 \%$ of $\mathrm{Mn}$ of $\mathrm{OEC}$ is $\mathrm{Mn}(\mathrm{II})$ at an electron

306 beam dose of 11.1 MGy used for the low-dose structure, the structure of the OEC retained

307 an occupancy of 1.0. This may be contributed by the stability of the structure of OEC, as

308 the metal ions of OEC are liganded by seven amino acid residues (D1-D170, D1-E189,

309 D1-H332, D1-E333, D1-D342, D1-A344, and CP43-E354). However, the longer

310 distances observed in some of the metal-oxygen distances of OEC even in the low-dose

311 structure indicated the existence of electron beam damage. Tanaka et al. has reported that,

312 using SR, a dose of 0.1 MGy is necessary to achieve a structure similar to that of the

313 XFEL structure ${ }^{29}$. Thus, in order to achieve a damage free structure, the electron beam

314 dose needs to be further reduced. Fortunately, our data indicated that the resolution

315 depends on the particles used, and by using more images and particles, it will be possible

316 to lower the electron beam dose and achieve the structure at a higher resolution.

317 Coordinate errors in the cryo-EM structure may be caused by the ambiguities in the

318 orientations of particles and their averaging, as well as the subsequent structural analysis

319 procedures. Structural analysis by cryo-EM at a higher resolution should eliminate such

320 errors, and gives rise to a more accurate structure. It is also expected that improvements in

321 the averaging and structural analysis algorithms of the cryo-EM data may improve the

322 accuracy of the structures at the same resolutions.

323 In summary, we show that the electron dose commonly used in cryo-EM is damaging

324 to protein samples. However, the damaged area was limited to redox-sensitive part. Our 
325 results suggest that it is possible to obtain a structure with less damage and high

326 resolution by reducing the total dose and increasing the number of particles. This study

327 will serve as a good indicator for determining damage-less cryo-EM structures of PSII

328 and all biological samples, especially redox-active metalloproteins.

\section{$331 \quad$ Methods}

332 Growth of cells and purification of PSII. Cells of Thermosynechococcus vulcanus (T.

333 vulcanus) were grown in four $10 \mathrm{~L}$ bottles at $50^{\circ} \mathrm{C}$. PSII with a high oxygen evolving

334 activity was purified from T. vulcanus as described previously $\mathrm{y}^{30-32}$ and suspended with a

335 buffer containing $20 \mathrm{mM}$ MES-NaOH (pH 6.0), 0.04\% $\beta$-dodecyl-D-maltopyranoside

336 and 5\% glycerol. For the Titan-96k and ARM-60k data collection, glycerol in the buffer

337 was removed by polyethylene glycol (PEG) precipitation and the resultant PSII was

338 re-suspended in a buffer containing $20 \mathrm{mM}$ MES-NaOH (pH 6.0), $20 \mathrm{mM} \mathrm{NaCl}, 3 \mathrm{mM}$

$339 \mathrm{CaCl}_{2}, 0.04 \% \quad \beta$-dodecyl-D-maltopyranoside.

341 Cryo-EM data collection. For cryo-EM experiments, $3-\mu \mathrm{L}$ aliquots of the PSII sample 342 at each condition (shown in Table 1) were applied to Quantifoil R1.2/1.3, Mo 300 mesh

343 or $\mathrm{Cu} 200$ mesh grids. The grids were incubated for $10 \mathrm{~s}$ in an FEI Vitrobot Mark IV at

$3444^{\circ} \mathrm{C}$ and $100 \%$ humidity. The grids were immediately plunged into liquid ethane cooled

345 by liquid nitrogen and then transferred into the Titan Krios (Thermo Fischer Scientific)

346 equipped with a field emission gun, a Cs corrector (CEOS GmbH), and a direct electron 
347 detection camera (Falcon 3EC, Thermo Fischer Scientific), or CRYO ARM 300 (JEOL)

348 equipped with a cold-field emission gun and a direct electron detection camera (Gatan K2

349 summit, Gatan Inc). These microscopes were operated at $300 \mathrm{kV}$ and a nominal

350 magnification of $\times 75,000$ (Titan-75k), $\times 96,000($ Titan-96k) for Titan Krios and $\times 60,000$

351 (ARM-60k) for CRYO ARM 300. Images were recorded using the Falcon 3EC in linear

352 mode or Gatan K2 summit in counting mode. Micrographs were recorded with a pixel

353 size of $0.870 \AA, 0.678 \AA$ and $0.822 \AA$ at a dose rate of 40 electrons $\AA^{-2} \mathrm{sec}^{-1}, 40$ electrons

$354 \AA^{-2} \sec ^{-1}$ and 83 electrons $\AA^{-2} \sec ^{-1}$ for Titan-75k, Titan-96k and ARM-60k, respectively.

355 The nominal defocus range were -1.0 to $-2.0 \mu \mathrm{m},-1.0$ to $-2.5 \mu \mathrm{m}$, and -0.8 to $-1.6 \mu \mathrm{m}$

356 for Titan-75k, Titan-96k and ARM-60k, respectively. Each exposure was conducted for

$35745.11 \mathrm{~s}, 26.64 \mathrm{~s}$ and $10.00 \mathrm{~s}$, and were dose-fractionated into 78, 39 and 50 movie frames

358 for Titan-75k, Titan-96k and ARM-60k, respectively. We acquired 2,084, 4,237 and

3592,160 images for the data sets of Titan-75k, Titan-96k and ARM-60k, respectively.

361 Cryo-EM image processing. Movie frames were aligned and summed using the

362 MotionCor2 software ${ }^{33}$ to obtain a final dose weighted image. Estimation of the contrast

363 transfer function (CTF) was performed using the CTFFIND4 program ${ }^{34}$. All of the

364 following processes were performed using RELION3.0 ${ }^{35}$. For structural analyses of the

365 Titan-75k data set, 354,233 particles were automatically picked from 2,084 micrographs

366 and then were used for reference-free 2D classification. Then, 309,028 particles were

367 selected from the good 2D classes and subjected to 3D classification with a C2 symmetry.

368 The $1.9 \AA$ A PSI structure from T. vulcanus (PDB: $3 \mathrm{WU} 2)^{6}$ was employed for the initial 
369 model for the first 3D classification with 60- $\AA$ low-pass filter. As shown in the

370 Supplementary Fig. 1 and 2, the PSII structure was reconstructed from 90,897 particles at

371 an overall resolution of $2.22 \AA$. For structural analyses of the Titan-96k data set, 612,287

372 particles were automatically picked from 4,237 micrographs and then used for

373 reference-free 2D classification. Then, 566,145 particles were selected from the good 2D

374 classes and subjected to 3D classification with a C2 symmetry. The 2.22- $\AA$ map from

375 Titan-75k data was employed for the initial model for the first 3D classification with a

$37660-\AA$ low-pass filter. As shown in the Supplementary Fig. 1 and 2, the PSII structure was

377 reconstructed from 203,912 particles at an overall resolution of $2.20 \AA$. For structural

378 analyses of the ARM-60k data set, 481,946 particles were automatically picked from

3792,160 micrographs and used for reference-free 2D classification. Then, 481,927 particles

380 were selected from the good $2 \mathrm{D}$ classes and subjected to $3 \mathrm{D}$ classification with a $\mathrm{C} 2$

381 symmetry. The 2.22- $\AA$ map from Titan-75k data was employed for the initial model for

382 the first $3 \mathrm{D}$ classification with a $60-\AA$ low-pass filter. The PSII structure was

383 reconstructed from 174,099 particles at an overall resolution of $1.95 \AA$ (Supplementary

384 Fig. 3 and 4). For the low-dose maps, the summing number of movie frames were

385 decreased in the final step of Bayesian polishing and used for reconstruction without

386 refinement of particle positions and orientations, using RELION ${ }^{35}$ with the command line

387 option "relion_reconstruct" and then post-processed in RELION ${ }^{35}$. All of the resolution

388 was estimated by the gold-standard Fourier shell correlation (FSC) curve with a cut-off

389 value of 0.143 (Supplementary Fig. 2 and 4$)^{36}$. The local resolution was estimated using

390 RELION $^{35}$. 
392 B-factor estimation. For the B-factor plot, the total set of all particles from the final

393 refinement was randomly resampled into smaller subsets. These subsets were subjected

394 to 3D auto-refinement and the resulting orientations were used to calculate

395 reconstructions for each of the two random halves used in the auto-refinement. The

396 squared values of the resulted, estimated resolutions were then plotted against the natural

397 logarithm of the number of particles in the subset, and B-factors were calculated from the

398 slope of the straight line best fitted with the points in the plot (Fig.1).

400 Model building and refinement. The $1.95-\AA$ and $2.08-\AA$ cryo-EM maps were used for

401 model building of the high-dose and low-dose PSII structures, respectively. First, the

402 crystal structure of T. vulcanus PSII (PDB: 3WU2) was manually fitted into each

403 cryo-EM map using UCSF Chimera ${ }^{37}$, and then the structures were inspected and

404 adjusted individually with COOT $^{38}$. The structures of high-dose PSII and low-dose PSII

405 were then refined with phenix.real_space_refine ${ }^{39}$ and Refmac5 $5^{40}$ with geometric

406 restraints for the protein-cofactor coordination. The positions of four manganese atoms

407 and one calcium atom were clearly visible in the cryo-EM map (Fig. 2 and 4). The

408 positions of the five oxo-oxygen atoms and four water molecules ligated to the OEC were

409 less clear, and they were identified by the difference map in which, the maps of metal ions

410 with a diameter of $1.5 \AA$ from that metal ion were subtracted from the whole cryo-EM

411 map, after placement of the manganese and calcium atoms (Fig. 2 and 4). The initial

412 positions of metal and oxygen atoms were assigned based on the highest peaks in the 
413 cryo-EM maps. This was taken as the initial structure. Subsequently, we performed the

414 structural refinement with loose restraints $(0.1 \sigma)$ for bond distances $(\mathrm{Mn}-\mathrm{O}$ and $\mathrm{Ca}-\mathrm{O})$

415 that were taken from the initial position. Then the refinement was performed with tighter

416 restraints $(0.05 \sigma)$ for bond distances successively using the modified 'new' library for the

417 bond distances. This geometry optimization procedure was repeated several times until

418 the bond distances converged. However, the distance of Mn4-O4 in the high-dose

419 structure, and the distances of Mn1-O1 and Mn3-O2 in the low-dose structure, were fixed

420 to $1.8 \AA$, because these distances were too close and could not be refined. The averages of

421 the distances of $\mathrm{Mn}-\mathrm{Mn}, \mathrm{Mn}-\mathrm{Ca}, \mathrm{Mn}-\mathrm{O}$ and $\mathrm{Mn}$-ligand were calculated from each PSIIs

422 in the final four refinement steps and are listed in Table 3. The final models were further

423 validated with Q-score ${ }^{41}$, MolProbity ${ }^{42}$ and EMringer $^{43}$. The statistics for all data

424 collection and structure refinement are summarized in Table 1 and 2. All structural

425 figures are made by Pymol $^{44}$ or UCSF ChimeraX ${ }^{45}$.

427 Difference map analysis between low-dose PSII and high-dose PSII. A difference

428 map was calculated by subtracting high-dose map from the low-dose map, i.e. (low-dose

429 PSII) minus (high-dose PSII). The rotational and translational matrix was calculated

430 based on the refined atomic coordinates using lsqkab in $\mathrm{CCP} 4^{46}$. A map of low-dose PSII

431 was superposed with a map of high-dose PSII which were applied by a low-pass filter and

432 adjusted to $2.08 \AA$ resolution, with calculated rotational and translational matrix using

433 maprot in $\mathrm{CCP} 4^{47}$. The high-dose PSII map and the low-dose PSII map was normalized

434 based on the ratio of the root mean square map density value and then the difference maps 
435 were calculated using UCSF Chimera ${ }^{37}$ with the command line option "vop subtract"

436 (Supplementary Fig. 8).

438 Data availability. Atomic coordinates and cyro-EM maps for the reported structure of

439 PSII determined from the high-dose data set of ARM-60K and low-dose data set of

440 ARM-60k were deposited in the Protein Data Bank under an accession codes 7D1T and

$4417 D 1 U$, respectively, and in the Electron Microscopy Data Bank under the accession codes

442 EMD-30547, EMD-30548, respectively. The cryo-EM maps of the Titan-75k data set and

443 Titan-96k data set were deposited in the Electron Microscopy Data Bank under the

444 accession codes EMD-30549 and EMD-30550, respectively.

\section{Acknowledgements}

448 This work was supported by JSPS KAKENHI No. JP20H02914 (K.K.), JP19K22396, 449 JP20H03194 (F.A.), JP20H05087 (N.M.), JP17H06434 (J.-R.S.), JST PRESTO No. 450 JPMJPR16P1 (F.A.), the Platform Project for Supporting Drug Discovery and Life

451 Science Research (Basis for Supporting Innovative Drug Discovery, Life Science 452 Research (BINDS)) of AMED No. JP18am0101072j002 (N.M.), and the Cyclic 453 Innovation for Clinical Empowerment (CiCLE) from AMED (K.Y.).

455 Author Contributions

456 J.-R.S. and K. Y. conceived the project; Y.N. and F.A. purified the PSII; N.M. and T.H. 
457 collected cryo-EM images; N. M., K.K. and T.H. processed the EM data. K.K. built the

458 structure model and refined the final models; K.K. analyzed the structure; and K.K., T.H.,

459 N.M. and J.-R.S. wrote the paper, and all of the authors joined the discussion of the

460 results.

461

462 


\section{References}

4641 Kok, B., Forbush, B., \& McGloin, M. Cooperation of charges in photosynthetic

465 oxygen evolution. I. A linear four step mechanism. Photochem. Photobiol. 11, $466 \quad 457-475(1970)$.

4672 Zouni, A. et al. Crystal structure of photosystem II from Synechococcus elongatus

$468 \quad 3.8$ A resolution. Nature 409, 739-743 (2001).

4693 Kamiya, N. \& Shen, J.-R. Crystal structure of oxygen-evolving photosystem II from

470 Thermosynechococcus vulcanus at 3.7-Å resolution. Proc. Natl Acad. Sci. USA 100,

$471 \quad 98-103(2003)$.

4724 Ferreira, K. N.et al. Architecture of the photosynthetic oxygen-evolving center.

$473 \quad$ Science 303, 1831-1838 (2004).

4745 Guskov, A. et al. Cyanobacterial photosystem II at 2.9- $\AA$ resolution and the role of 475 quinones, lipids, channels and chloride. Nat. Struct. Mol. Biol. 16, 334-342 (2009).

4766 Umena, Y. et al. Crystal structure of oxygen-evolving photosystem II at a resolution 477 of 1.9 A. Nature $473,55-60$ (2011).

4787 Yano, J. et al. X-ray damage to the Mn4Ca complex in single crystals of 479 photosystem II: a case study for metalloprotein crystallography. Proc. Natl Acad. $480 \quad$ Sci. USA 102, 12047-12052 (2005).

4818 Neutze, R. et al. Potential for biomolecular imaging with femtosecond X-ray pulses, $482 \quad$ Nature, 406, $752-757$ (2000).

4839 Hirata, K. et al. A $1.9 \AA$ resolution structure of $420 \mathrm{kDa}$ cytochrome oxidase by 484 femtosecond crystallography. Nat. Methods 11, 734-736 (2014). 
48510 Suga, M. et al. Native structure of photosystem II at $1.95 \mathrm{~A} \square$ resolution viewed by

486 femtosecond X-ray pulses. Nature 517, 99-103 (2015).

48711 Suga, M. et al. Light-induced structural changes and the site of $\mathrm{O}=\mathrm{O}$ bond

488 formation in PSII caught by XFEL. Nature 543, 131-135 (2017).

48912 Kern, J. et al. Structures of the intermediates of Kok's photosynthetic water

$490 \quad$ oxidation clock. Nature 563, 421-425. (2018).

49113 Suga, M. et al. An oxyl/oxo mechanism for oxygen-oxygen coupling in PSII

492 revealed by an X-ray free-electron laser. Science 366, 334-338 (2019).

49314 Ibrahim, M. et al. Untangling the sequence of events during the S2 $\rightarrow$ S3 transition

494 in photosystem II and implications for the water oxidation mechanism. Proc. Natl

$495 \quad$ Acad. Sci. USA 117, 12624-12635 (2020).

49615 Kato, Y. et al. Fourier transform infrared analysis of the S-state cycle of water

497 oxidation in the microcrystals of photosystem II. J. Phys. Chem. Lett. 9, 2121-2126

$498 \quad$ (2018).

49916 Flores, J.A. et al. Connexin-46/50 in a dynamic lipid environment resolved by

$500 \quad$ Cryo-EM at $1.9 \AA$ A. Nature communications 11, 4331 (2020).

50117 Nakane, T. et al. Single-particle cryo-EM at atomic resolution. Preprint at $502 \quad$ https://doi.org/10.1101/2020.05.22.110189 (2020).

50318 Yip, K.M. et al. Breaking the next Cryo-EM resolution barrier - Atomic resolution 504 determination of protein! Preprint at https://doi.org/10.1101/2020.05.21.106740 $505 \quad$ (2020). 
50619 Burmeister, W.P. Structural changes in a cryo-cooled protein crystal owing to radi-

$507 \quad$ ation damage. Acta Crystal. D 56, 328-341 (2000).

50820 Ravelli, R.B. et al. The "fingerprint" that X-rays can leave on structures. Structure

$509 \quad \mathbf{8}, 315-328(2000)$.

51021 Weik, M. et al. Specific chemical and structural damage to proteins produced by

511 synchrotron radiation. Proc. Natl. Acad. Sci. U.S.A. 97, 623-628 (2000).

51222 Hamaguchi, T., Maki-Yonekura, S., Naitow, H., Matsuura, Y., Ishikawa, T. and

513 Yonekura, K. A new cryo-EM system for single particle analysis. J. Struct. Biol.

$514 \quad 207,40-48(2019)$.

51523 Rosenthal, P. B. and Henderson, R., Optimal determination of particle orientation,

516 absolute hand, and contrast loss in single-particle electron cryomicroscopy. J. Mol.

$517 \quad$ Biol. 333, 721-45 (2003).

51824 Koua, F.H.M., Umena, Y., Kawakami K. \& Shen J.-R. Structure of Sr-substituted

519 photosystem II at $2.1 \AA$ resolution and its implications in the mechanism of water

$520 \quad$ oxidation. Proc. Natl. Acad. Sci. USA 110, 3889-3894 (2013).

52125 Wang, J. Et al. Reduced occupancy of the oxygen-evolving complex of

522 photosystem II detected in cryo-electron microscopy maps. Biochemistry 57, $523 \quad 5925-5929(2018)$.

52426 Yonekura, K. Electron crystallography of ultrathin 3D protein crystals: Atomic 525 model with charges. Proc. Natl Acad. Sci. USA 112, 3368-3373 (2015).

52627 Baker, L. A. Et al. Radiation damage in electron cryomicroscopy. Methods in $527 \quad$ Enzym. 481, 371-388 (2010). 
52828 Henderson, R. Cryo-protection of protein crystals against radiation damage in

529 electron and X-Ray diffraction. Proc. R. Soc. Lond. B 241, 6-8 (1990).

53029 Tanaka, A. et al. Two different structures of the oxygen-evolving complex in the

531 same polypeptide frameworks of photosystem II J. Am. Chem. Soc. 139, 1728-1721

$532 \quad$ (2017).

53330 Shen, J.-R., and Inoue, Y. Binding and functional properties of two new extrinsic

534 components, cytochrome $c-550$ and a $12 \mathrm{kDa}$ protein, in cyanobacterial

535 photosystem II. Biochemistry 32, 1825-1832 (1993).

53631 Shen, J.-R., and Kamiya, N. Crystallization and the crystal properties of the

537 oxygen-evolving photosystem II from Synechococcus vulcanus. Biochemistry 39,

$538 \quad 14739-14744(2000)$.

53932 Shen, J.-R. et al. Purification and crystallization of oxygen-evolving photosystem II

540 core complex from thermophilic cyanobacteria. Methods in Mol. Biol. 684, 41-51

$541 \quad$ (2011).

54233 Zheng, S.Q. et al. MotionCor2: anisotropic correction of beam-induced motion for

543 improved cryo-electron microscopy. Nat. Methods 14, 331-332 (2017).

54434 Mindell, J.A., and Grigorieff, N. Accurate determination of local defocus and 545 specimen tilt in electron microscopy. J Struct. Biol. 142, 334-347 (2003).

54635 Scheres, S.H. RELION: implementation of a Bayesian approach to cryo-EM $547 \quad$ structure determination. J. Struct. Biol. 180, 519-530 (2012).

54836 Grigorieff, N., and Harrison, S.C. Near-atomic resolution reconstructions of 549 icosahedral viruses from electron cryo-microscopy. Curr. Opin. Struct. Biol. 21, 
$550 \quad 265-273(2011)$

55137 Pettersen, E. F. et al. UCSF Chimera - a visualization system for exploratory

552 research and analysis. J. Comp. Chem. 25, 1605-1612 (2004).

55338 Emsley, P. et al. Features and development of Coot. Acta Crystal. D 66, 486-501

$554 \quad$ (2010).

55539 Adams, P. D. et al. PHENIX: a comprehensive Python-based system for 556 macromolecular structure solution. Acta Crystal. D 66, 213-221 (2010).

55740 Murshudov, G. N. et al. REFMAC5 for the refinement of macromolecular crystal $558 \quad$ structures. Acta Crystal. D 67, 355-367 (2011).

55941 Pintilie, G. et.al. Measurement of atom resolvability in cryo-EM maps with

560 Q-scores. Nat. Methods 17, 328-334 (2020).

56142 Chen, V. B. et al. MolProbity: all-atom structure validation for macromolecular 562 crystallography. Acta Crystal. D 66, 12-21 (2010).

56343 Barad, B. A. et al. EMRinger: side chain-directed model and map validation for 3D

564 cryo-electron microscopy. Nat. Methods 12, 943 (2015).

56544 The PyMOL Molecular Graphics System, Version 2.1, Schrödinger, LLC.

56645 Goddard, T.D. et al. UCSF ChimeraX: Meeting modern challenges in visualization

$567 \quad$ and analysis. Protein Sci. 27, 14-25 (2018).

56846 Winn, M. D. et al. Overview of the CCP4 suite and current developments. Acta $569 \quad$ Crystal. D 67, 235-242 (2011).

57047 Kato K. et al. Structural basis for the adaptation and function of chlorophyll $f$ in 571 photosystem I. Nat. Commun. 11, 1-10 (2020). 


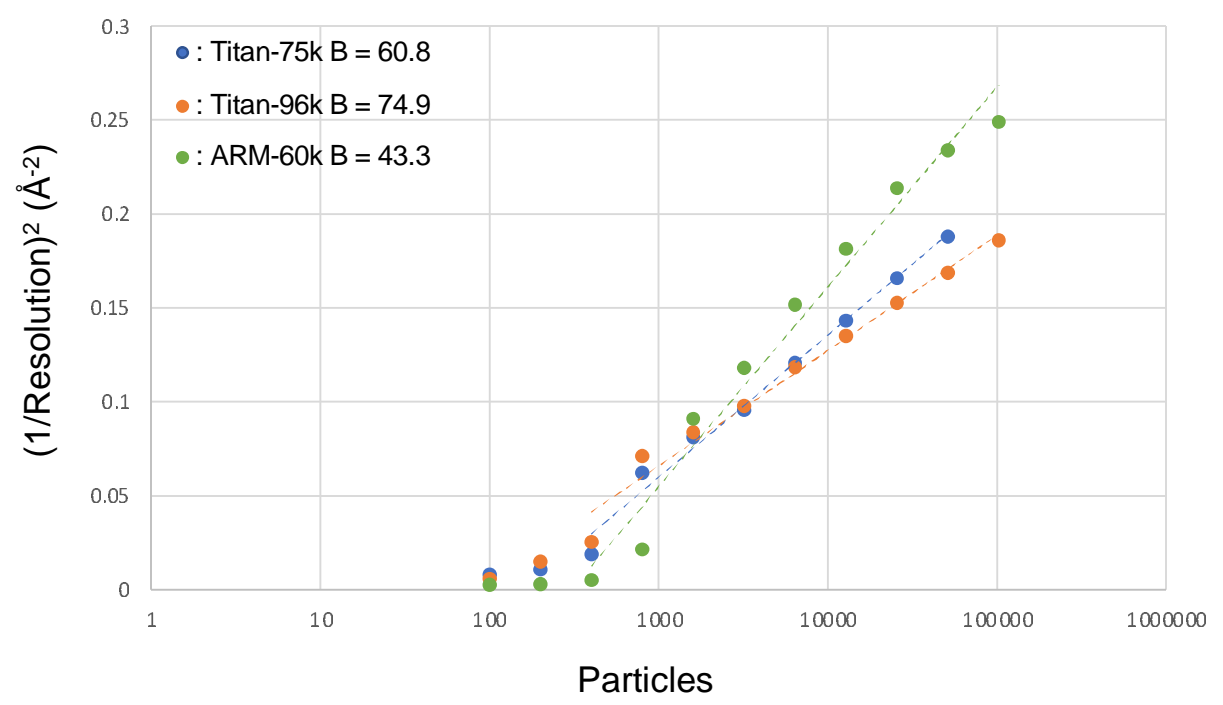

57248

57349 Fig. 1 B-factor plot for the data sets of Titan-75k, Titan-96k and ARM-60k. B

574 factor plot for the Titan-75k data set at a dose of $40 \mathrm{e}^{-} \AA^{-2}$ (blue), the Titan-96k data

575 set at a dose of $40 \mathrm{e}^{-} \AA^{-2}$ (orange), and the ARM-60k data set at a dose of $83 \mathrm{e}^{-} \AA^{-2}$

576 (green).

$577 \quad 50$ 
a

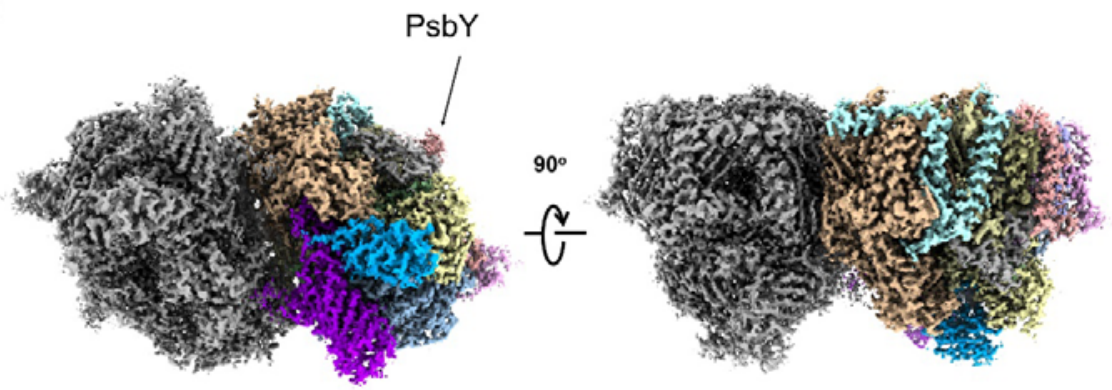

b
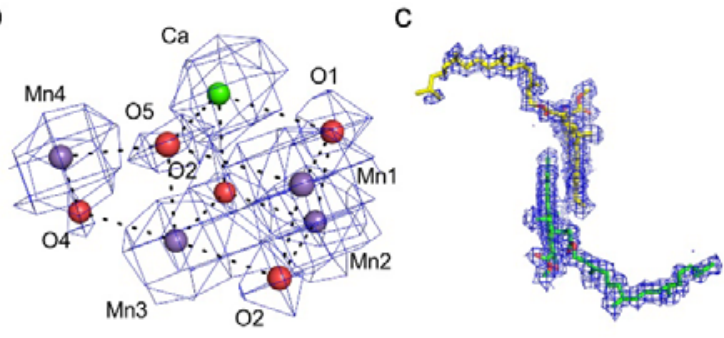

d

e
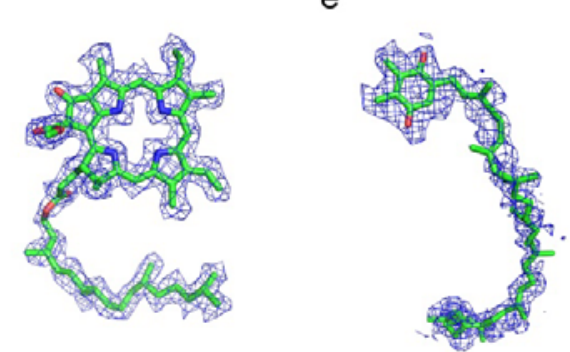

f

579 Fig. 2 Overall structure of PSII at a high-dose. a The cryo-EM density of the PSII at

$5801.95 \AA$ resolution from the ARM-60k data set. b-e The cryo-EM density of cofactors,

581 OEC (b), P680 (c), pheophytin (d) and plastoquinone $\left(\mathrm{Q}_{\mathrm{B}}\right)(\mathbf{e})$, superposed with the

582 refined model. $\mathbf{f}$ The density of PsbY superposed with the refined model. The densities 583 were depicted at $5 \sigma$. 
a

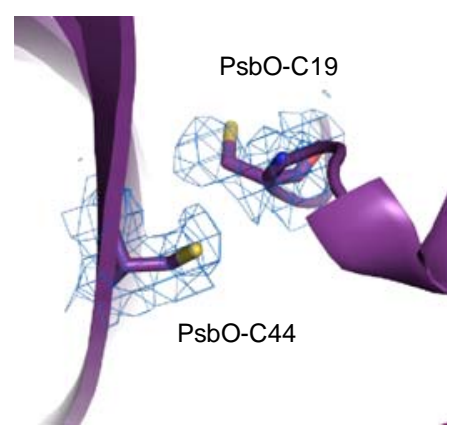

b

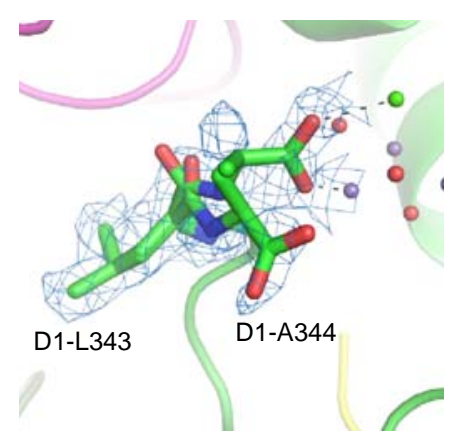

C

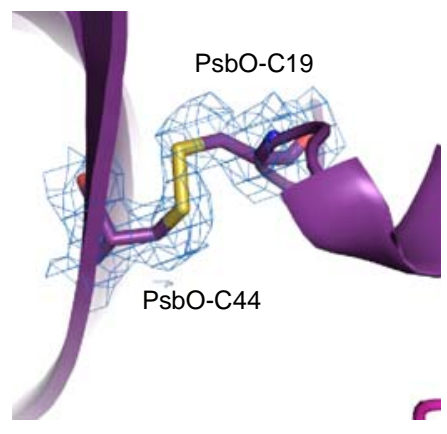

d

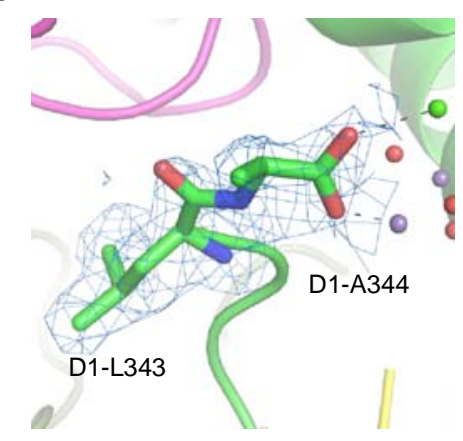

587 Fig. 3 Electron beam damages in the PSII structure of the ARM-60k data set at the

588 high-dose $\left(83 \mathbf{e}^{-} \AA^{-2}\right)$ and low-dose $\left(3.3 \mathbf{e}^{-} \AA^{-2}\right)$. a The broken disulfide bond in PsbO at

589 the high-dose. b The alternative conformation at D1-A344 at the high dose. c The

590 disulfide bond recovered in PsbO at the low-dose. d The single conformation of

591 D1-A344 at the low dose. The densities were depicted at $5 \sigma$.

592 
a

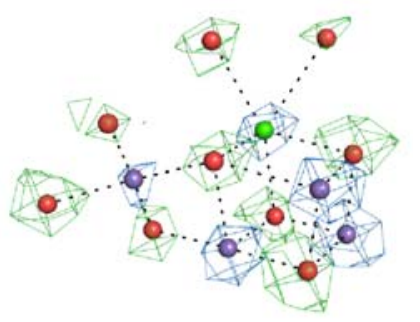

b

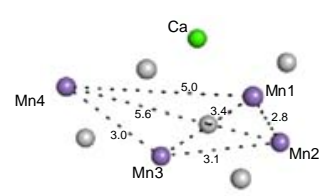

C
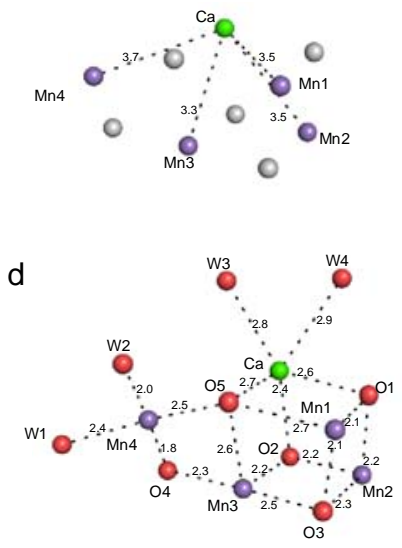

e

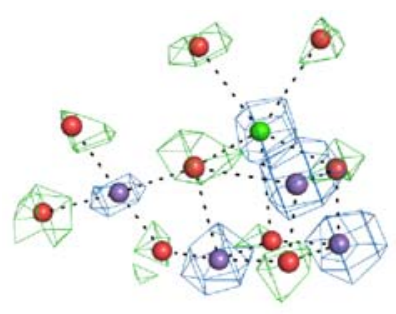

f

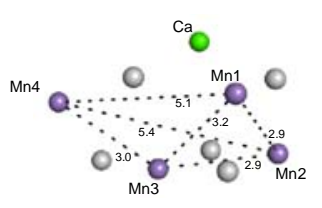

g

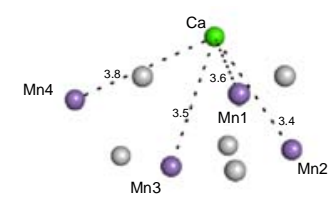

h

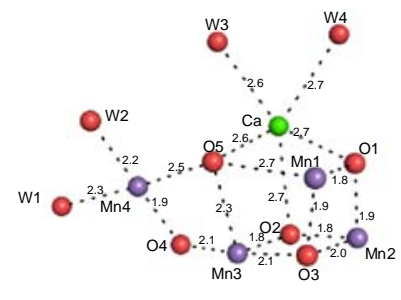

594

595 Fig. 4 Electron beam damages in the OEC structure solved at the high-dose $\left(83 \mathrm{e}^{-}\right.$

$596 \AA^{-2}$ ) and low-dose $\left(3.3 \mathbf{e}^{-} \AA^{-2}\right)$. a-d: High dose structure. a The cryo-EM density (blue)

597 for manganese and calcium atoms at $17 \sigma$ and the subtracted map (green) for oxygen

598 atoms and water molecules at 7 б. b Mn-Mn distances in the OEC (in $\AA$ ). c Mn-Ca

599 distances in the OEC (in $\AA$ ) d Mn-O, Ca-O, Mn-water and $\mathrm{Ca}$-water distances in the

600 OEC (in $\AA$ ). e-h: Low dose structure. e The cryo-EM density (blue) for manganese and

601 calcium atoms at $17 \sigma$ and the subtracted map (green) for oxygen atoms and water 
603 (in $\AA$ ). $\mathbf{h ~ M n - O , C a - O , ~ M n - w a t e r ~ a n d ~} \mathrm{Ca}$-water distances in the OEC (in $\AA$ ).
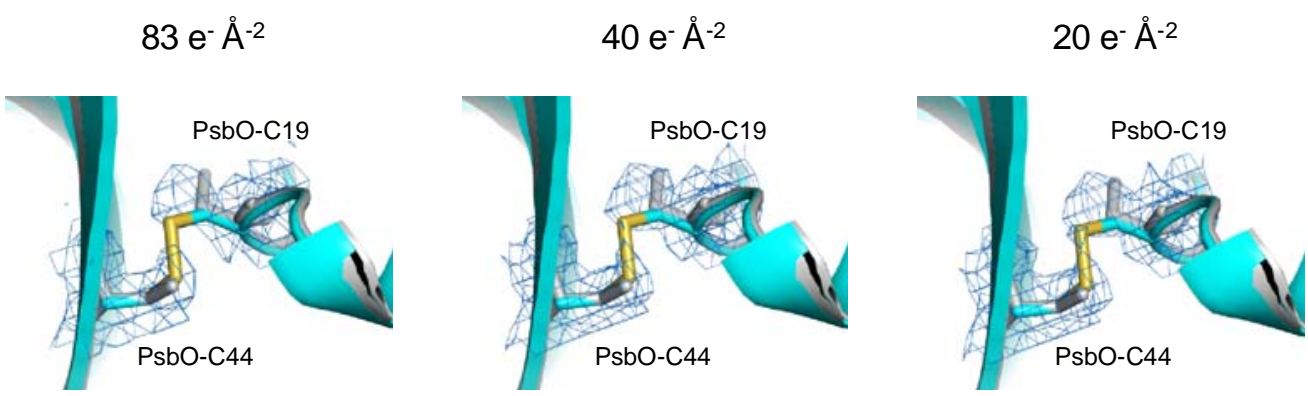

$10 e^{-} \AA^{-2}$

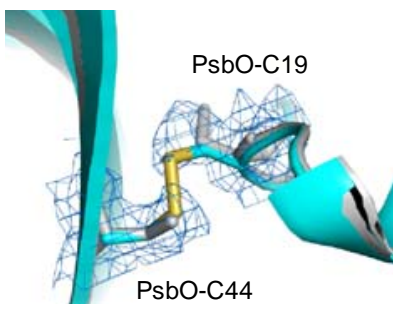

$5 e^{-} \AA^{-2}$

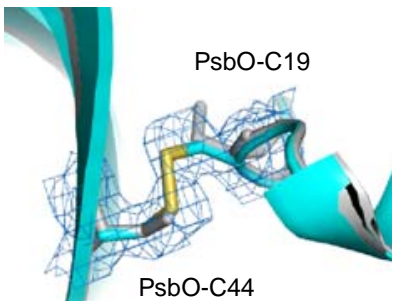

$3.3 e^{-} \AA^{-2}$

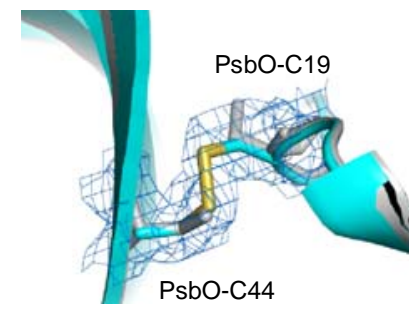

604

605 Fig. 5 Changes of the cryo-EM map in the region of the disulfide bond in PsaO

606 with changes of the electron beam dose. The cryo-EM maps for each electron dose are

607 displayed as a blue mesh at $4 \sigma$ and the corresponding models for low-dose (colored)

608 and high-dose (gray) are shown as sticks.

609 
610 Table 1 Sample preparation and Cryo-EM data collection parameters.

\begin{tabular}{|c|c|c|c|}
\hline Data set & Titan-75K & Titan-96K & ARM-60K \\
\hline $\begin{array}{l}\text { Original sample } \\
\text { conc. }\end{array}$ & $3.8 \mathrm{Chl} \mathrm{mg/ml}$ & $9.7 \mathrm{Chl} \mathrm{mg/ml}$ & $9.7 \mathrm{Chl} \mathrm{mg/ml}$ \\
\hline $\begin{array}{c}\text { Original sample } \\
\text { buffer }\end{array}$ & $\begin{array}{c}20 \mathrm{mM} \text { MES (pH6.0), 5\% } \\
\text { glycerol, } 0.04 \% \beta-\mathrm{DDM}\end{array}$ & $\begin{array}{c}20 \text { mM MES } \\
(\mathrm{pH} 6.0)\end{array}$ & $\begin{array}{c}20 \text { mM MES } \\
(\mathrm{pH} 6.0)\end{array}$ \\
\hline Dilution buffer & $\begin{array}{c}20 \mathrm{mM} \text { MES (pH6.0), } \\
0.04 \% \beta-\mathrm{DDM}\end{array}$ & $\begin{array}{c}20 \mathrm{mM} \text { MES } \\
(\mathrm{pH} 6.0)\end{array}$ & $\begin{array}{c}20 \text { mM MES } \\
(\mathrm{pH} 6.0)\end{array}$ \\
\hline Dilution & 10-fold & 5-fold & 5-fold \\
\hline Final sample conc. & $0.38 \mathrm{Chl} \mathrm{mg/ml}$ & $1.94 \mathrm{Chl} \mathrm{mg/ml}$ & $1.94 \mathrm{Chl} \mathrm{mg/ml}$ \\
\hline \multicolumn{4}{|l|}{$\begin{array}{c}\text { Data collection and } \\
\text { processing }\end{array}$} \\
\hline Microscope & Titan Krios & Titan Krios & CRYO ARM 300 \\
\hline Detector & $\begin{array}{l}\text { Falcon3EC } \\
\text { in EC mode }\end{array}$ & $\begin{array}{l}\text { Falcon3EC } \\
\text { in EC mode }\end{array}$ & $\begin{array}{l}\text { Gatan K2 summit } \\
\text { in Counting mode }\end{array}$ \\
\hline Magnification & $75 \mathrm{~K}$ & $96 \mathrm{~K}$ & $60 \mathrm{~K}$ \\
\hline Voltage $(\mathrm{kV})$ & 300 & 300 & 300 \\
\hline $\begin{array}{l}\text { Defocus range } \\
\qquad(\mu \mathrm{m})\end{array}$ & -1.00 to -2.00 & -1.00 to -2.50 & -0.8 to -1.6 \\
\hline Pixcel size $(\AA)$ & 0.870 & 0.678 & 0.822 \\
\hline $\begin{array}{l}\text { Total electron dose } \\
\qquad\left(\AA^{2}\right)\end{array}$ & 40 & 40 & 83 \\
\hline Exposure time (s) & 45.11 & 24.64 & 10 \\
\hline $\begin{array}{c}\text { Number of frames } \\
\text { per image }\end{array}$ & 78 & 39 & 50 \\
\hline $\begin{array}{l}\text { Number of } \\
\text { micrographs }\end{array}$ & 2,084 & 4,237 & 2,160 \\
\hline $\begin{array}{l}\text { Initial particle } \\
\text { images }\end{array}$ & 354,233 & 612,287 & 444,729 \\
\hline $\begin{array}{l}\text { Final particle } \\
\text { images }\end{array}$ & 90,897 & 203,912 & 174,099 \\
\hline Map resolution $(\AA)$ & $2.23(2.22)^{*}$ & $2.26(2.20)^{*}$ & $1.98(1.95)^{*}$ \\
\hline $\begin{array}{c}\text { Map sharpening } \\
\text { B-factor }\left(\AA^{2}\right)\end{array}$ & $-54(-53)^{*}$ & $-60(-56)^{*}$ & $-34(-32)^{*}$ \\
\hline $\begin{array}{l}\text { Rosenthal-Henders } \\
\text { on B factor }\left(\AA^{2}\right)\end{array}$ & 60.8 & 74.9 & 43.3 \\
\hline Applied symmetry & $\mathrm{C} 2$ & $\mathrm{C} 2$ & $\mathrm{C} 2$ \\
\hline
\end{tabular}


613 Table 2 Statistics of data collection, processing and refinement.

614

\begin{tabular}{|c|c|c|}
\hline Refinement & High-dose & Low-dose \\
\hline PDB ID & 7D1T & 7D1U \\
\hline EMDB ID & EMD-30547 & EMD-30548 \\
\hline Initial Model used (PDB code) & $3 \mathrm{WU} 2$ & $3 \mathrm{WU} 2$ \\
\hline Model resolution $(\AA)$ & 1.94 & 2.03 \\
\hline FSC threshold & 0.5 & 0.5 \\
\hline \multicolumn{3}{|l|}{ No. of atoms } \\
\hline Protein & 41708 & 41680 \\
\hline Ligand & 8854 & 8854 \\
\hline Water & 2432 & 2121 \\
\hline \multicolumn{3}{|l|}{ B factors $\left(\AA^{2}\right)$} \\
\hline Protein & 20.6 & 21.2 \\
\hline Ligand & 23.5 & 24.1 \\
\hline Water & 26.4 & 23.7 \\
\hline \multicolumn{3}{|l|}{ R.m.s deviations } \\
\hline Bond lengths $(\AA)$ & 0.012 & 0.010 \\
\hline Bond angles $\left(^{\circ}\right)$ & 1.46 & 1.31 \\
\hline \multicolumn{3}{|l|}{ Validation } \\
\hline MolProbity score & 1.3 & 1.5 \\
\hline Clashscore & 1.8 & 2.2 \\
\hline Poor rotamers $(\%)$ & 2.4 & 3.1 \\
\hline EMRinger score & 7.0 & 6.8 \\
\hline Q-Score & 0.87 & 0.86 \\
\hline \multicolumn{3}{|l|}{ Ramachandran plot } \\
\hline Favored $(\%)$ & 97.93 & 97.55 \\
\hline Allowd (\%) & 1.88 & 2.26 \\
\hline Disallowed (\%) & 0.19 & 0.19 \\
\hline
\end{tabular}

615

616

617

618

619 
620 Table 3 Summarization of the distances of atoms of the $\mathrm{Mn}_{4} \mathrm{CaO}_{5}$ cluster.

\begin{tabular}{|c|c|c|c|c|c|c|c|c|c|c|c|c|c|c|}
\hline & $\begin{array}{c}\text { High- } \\
\text { dose }\end{array}$ & $\begin{array}{l}\text { Low- } \\
\text { dose }\end{array}$ & $\begin{array}{c}\text { SR } \\
(3 W U 2)\end{array}$ & $\begin{array}{c}\text { XFEL } \\
\text { (4UB6 }\end{array}$ & & & ligh-do & & & & & ow-do & ose & \\
\hline & $\begin{array}{l}\text { (Aver } \\
\text {-age) }\end{array}$ & $\begin{array}{l}\text { (Aver } \\
\text {-age) }\end{array}$ & $\begin{array}{l}\text { (Aver } \\
\text {-age) }\end{array}$ & $\begin{array}{l}(\mathrm{Av} \\
\text { er-a } \\
\text { ge })\end{array}$ & 4th & $3 \mathrm{rd}$ & 2nd & $1 \mathrm{st}$ & $\begin{array}{c}\text { Init- } \\
\text { ial }\end{array}$ & 4th & $3 \mathrm{rd}$ & 2nd & $1 \mathrm{st}$ & $\begin{array}{l}\text { Init- } \\
\text { ial }\end{array}$ \\
\hline Mn1-Mn2 & 2.8 & 2.9 & 2.8 & 2.7 & 2.81 & 2.83 & 2.82 & 2.82 & 2.81 & 2.88 & 2.87 & 2.89 & 2.8 & 2.95 \\
\hline Mn1-Mn3 & 3.4 & 3.2 & 3.3 & 3.2 & 3.35 & 3.36 & 3.35 & 3.36 & 3.46 & 3.20 & 3.20 & 3.24 & 3.18 & 3.37 \\
\hline Mn1-Mn4 & 5.0 & 5.1 & 5 & 5 & 5.04 & 5.02 & 5.03 & 5.03 & 4.99 & 5.10 & 5.09 & 5.08 & 5.04 & 5.32 \\
\hline Mn2-Mn3 & 3.1 & 2.9 & 2.9 & 2.7 & 3.08 & 3.10 & 3.09 & 3.09 & 3.06 & 2.88 & 2.88 & 2.88 & 2.85 & 2.75 \\
\hline Mn2-Mn4 & 5.6 & 5.4 & 5.4 & 5.2 & 5.65 & 5.65 & 5.65 & 5.63 & 5.42 & 5.47 & 5.46 & 5.42 & 5.43 & 5.40 \\
\hline Mn3-Mn4 & 3.0 & 3.0 & 3.0 & 2.9 & 2.97 & 2.97 & 2.98 & 2.94 & 2.67 & 3.04 & 3.03 & 3.01 & 3.00 & 3.05 \\
\hline Mn1-Ca & 3.5 & 3.6 & 3.5 & 3.5 & 3.55 & 3.52 & 3.52 & 3.52 & 3.51 & 3.59 & 3.57 & 3.55 & 3.56 & 3.83 \\
\hline Mn2-Ca & 3.5 & 3.4 & 3.4 & 3.3 & 3.49 & 3.45 & 3.46 & 3.46 & 3.29 & 3.44 & 3.43 & 3.41 & 3.44 & 3.35 \\
\hline Mn3-Ca & 3.3 & 3.5 & 3.4 & 3.4 & 3.32 & 3.29 & 3.28 & 3.27 & 3.04 & 3.45 & 3.45 & 3.44 & 3.46 & 3.43 \\
\hline Mn4-Ca & 3.7 & 3.8 & 3.8 & 3.8 & 3.73 & 3.69 & 3.70 & 3.68 & 3.57 & 3.76 & 3.76 & 3.70 & 3.80 & 3.86 \\
\hline Mn1-O1 & 2.1 & 1.8 & 1.9 & 1.8 & 2.11 & 2.10 & 2.08 & 2.06 & 2.34 & 1.80 & 1.76 & 1.77 & 1.78 & 2.09 \\
\hline Mn1-O3 & 2.1 & 1.9 & 1.8 & 1.9 & 2.09 & 2.10 & 2.10 & 2.09 & 2.29 & 1.89 & 1.90 & 1.91 & 1.92 & 1.90 \\
\hline Mn1-O5 & 2.7 & 2.7 & 2.6 & 2.7 & 2.72 & 2.73 & 2.74 & 2.75 & 2.64 & 2.71 & 2.72 & 2.72 & 2.72 & 2.89 \\
\hline Mn2-O1 & 2.2 & 1.9 & 2.1 & 1.8 & 2.18 & 2.17 & 2.17 & 2.16 & 2.19 & 1.98 & 1.95 & 1.92 & 1.86 & 2.12 \\
\hline Mn2-O2 & 2.2 & 1.8 & 2.1 & 1.8 & 2.24 & 2.23 & 2.2 & 2.17 & 2.45 & 1.84 & 1.83 & 1.82 & 1.80 & 2.45 \\
\hline Mn2-O3 & 2.3 & 2.0 & 2.1 & 2.0 & 2.28 & 2.28 & 2.27 & 2.26 & 2.36 & 2.01 & 2.01 & 2.01 & 2.00 & 1.95 \\
\hline Mn3-O2 & 2.2 & 1.8 & 1.9 & 1.9 & 2.18 & 2.18 & 2.16 & 2.14 & 2.04 & 1.80 & 1.78 & 1.81 & 1.86 & 2.08 \\
\hline Mn3-O3 & 2.5 & 2.1 & 2.1 & 2.1 & 2.48 & 2.47 & 2.44 & 2.42 & 2.48 & 2.05 & 2.06 & 2.06 & 2.07 & 2.02 \\
\hline Mn3-O4 & 2.3 & 2.1 & 2.1 & 1.9 & 2.30 & 2.30 & 2.28 & 2.26 & 2.38 & 2.17 & 2.11 & 2.05 & 1.96 & 2.71 \\
\hline Mn3-O5 & 2.6 & 2.3 & 2.4 & 2.2 & 2.58 & 2.58 & 2.56 & 2.54 & 2.58 & 2.35 & 2.32 & 2.29 & 2.23 & 2.57 \\
\hline Mn4-O4 & 1.8 & 1.9 & 2.1 & 2.0 & 1.80 & 1.80 & 1.79 & 1.82 & 1.96 & 1.86 & 1.89 & 1.92 & 1.97 & 2.05 \\
\hline Mn4-05 & 2.5 & 2.5 & 2.5 & 2.3 & 2.49 & 2.46 & 2.45 & 2.44 & 2.59 & 2.5 & 2.48 & 2.45 & 2.40 & 2.67 \\
\hline Mn4-W1 & 2.4 & 2.3 & 2.2 & 2.3 & 2.38 & 2.40 & 2.39 & 2.40 & 2.54 & 2.31 & 2.32 & 2.36 & 2.32 & 2.33 \\
\hline Mn4-W2 & 2.0 & 2.2 & 2.2 & 2.1 & 2.05 & 2.03 & 2.03 & 2.07 & 2.11 & 2.18 & 2.18 & 2.18 & 2.15 & 1.84 \\
\hline Ca-O1 & 2.6 & 2.7 & 2.4 & 2.6 & 2.63 & 2.63 & 2.62 & 2.62 & 2.60 & 2.72 & 2.70 & 2.67 & 2.63 & 2.47 \\
\hline Ca-O2 & 2.4 & 2.7 & 2.5 & 2.7 & 2.39 & 2.39 & 2.41 & 2.43 & 2.41 & 2.67 & 2.68 & 2.69 & 2.71 & 2.73 \\
\hline Ca-O5 & 2.7 & 2.6 & 2.7 & 2.5 & 2.71 & 2.70 & 2.68 & 2.66 & 2.70 & 2.62 & 2.60 & 2.58 & 2.55 & 2.60 \\
\hline Ca-W3 & 2.8 & 2.6 & 2.4 & 2.6 & 2.76 & 2.79 & 2.78 & 2.78 & 2.80 & 2.56 & 2.56 & 2.57 & 2.53 & 2.64 \\
\hline Ca-W4 & 2.9 & 2.7 & 2.5 & 2.5 & 2.89 & 2.93 & 2.92 & 2.94 & 2.92 & 2.71 & 2.71 & 2.73 & 2.65 & 2.71 \\
\hline
\end{tabular}

621 